\title{
Role of Octreotide in Pediatric Gastrointestinal Bleeding Secondary to Angiodysplasia in Children With Right Heart Failure
}

\author{
Kanika Puri; Randall L. Caldwell; Jean P. Molleston
}

\begin{abstract}
Objectives: Angiodysplasia is a relatively uncommon cause of GI bleeding in children and may be seen in right heart failure. Octreotide has been used successfully in adult patients with gastrointestinal bleeding due to angiodysplasias.

Methods: We describe two patients who had congenital heart disease with right heart failure and gastrointestinal bleeding from angiodysplasia.

Results: Angiodysplasia lesions were documented on traditional endoscopy and capsule endoscopy. Bleeding resolved after initiation of IV octreotide and did not recur on subcutaneous octreotide during the two year follow up period.

Conclusions: Based on the successful outcomes in the two patients, a trial of octreotide may be considered in pediatric patients who present with gastrointestinal bleeding secondary to angiodysplasia.
\end{abstract}

Keywords: angiodysplasia; gastrointestinal bleeding; octreotide; pediatric

Abbreviations: AD, Angiodysplasia; vWD, von Willebrand disease; IRB, Institutional Review Board; Hb, Hemoglobin; GAVE, gastric antral vascular ectasia; APC, Argon Plasma Coagulation; DBE, Double balloon Enteroscopy

This is the author's manuscript of the article published in final edited form as:

Puri, K., Caldwell, R., \& Molleston, J. P. (2017). Role of Octreotide in Pediatric Gastrointestinal Bleeding Secondary to Angiodysplasia in Children with Right Heart Failure. Journal of pediatric gastroenterology and nutrition, 66(2):e41-e44. https://doi.org/10.1097/MPG.0000000000001659 


\section{What is known:}

1. Angiodysplasias can cause gastrointestinal bleeding.

2. Octreotide has been used successfully to help control gastrointestinal bleeding in adult patients with variceal bleeding.

\section{What is new:}

1. Identification of angiodysplasia as source of significant GI bleeding in children with right heart failure.

2. Utilization of octreotide for control of bleeding from angiodysplasias. 


\section{Introduction}

Angiodysplasias (AD) are the most common vascular anomalies of the gastrointestinal tract. These are the second most common cause of gastrointestinal bleeding in adults. There is higher prevalence of $\mathrm{AD}$ in patients with end-stage renal disease, von Willebrand disease (vWD), and adults with aortic stenosis (Heyde's syndrome). We describe 2 patients with right heart failure who presented with gastrointestinal bleeding due to angiodysplasias which was treated with octreotide.

\section{Methods:}

This study was a retrospective review of the electronic medical records of two pediatric patients with right failure and gastrointestinal bleeding at Riley Hospital for Children at Indiana University Health. The study was approved by the Institutional Review Board (IRB).

\section{Results:}

A 17-year-old female (Patient A) with pulmonary stenosis, tricuspid atresia, hypoplastic right ventricle status post Fontan procedure, and protein losing enteropathy presented at age 14 years with anemia (Hb 7-8g/dL). Her hemoglobin dropped (from baseline of $16 \mathrm{~g} / \mathrm{dL}$ ) to 5.1g/dL. She required four units of blood. CT angiogram did not reveal any vascular malformation. She underwent upper endoscopy and colonoscopy which revealed vascular ectasias within the second part of the duodenum (Figure 1). Capsule endoscopy showed active bleeding attributed to angioectasias and small clots throughout the small bowel, especially in the terminal ileum . As she continued to bleed, she was started on octreotide with a bolus of $1 \mathrm{mcg} / \mathrm{kg}$ followed by a continuous infusion at the rate of $1 \mathrm{mcg} / \mathrm{kg} / \mathrm{hr}$ for 60 hours and then switched to $50 \mathrm{mcg}$ subcutaneous injections thrice daily for 14 days. The gastrointestinal bleeding resolved and her hemoglobin stabilized (Figure 2). She was then transitioned to octreotide $10 \mathrm{mg}$ monthly depot octreotide injections. She did not have any further hematochezia episodes during her treatment over a period of 2 years. She later developed asymptomatic vitamin B12 deficiency. The medication was discontinued uneventfully after 2 years with stable hemoglobin.

A 14-year-old male (Patient B) with hypoplastic left heart syndrome status post Fontan and Norwood procedures, portal hypertension, cirrhosis and ascites developed bloody stools and a drop in hemoglobin to $8.7 \mathrm{~g} / \mathrm{dL}$. He underwent an emergent endoscopy with flexible sigmoidoscopy. No abnormalities were found. A Meckel's scan was negative. Capsule endoscopy showed blood in the ileocecal region although no discrete lesions were noted. He underwent colonoscopy which showed erythema in cecum and terminal ileum. Biopsies demonstrated dilated blood vessels filled with red blood cells, consistent with vascular ectasias (Figure 3). He received a bolus of $1 \mathrm{mcg} / \mathrm{kg}$ of octreotide followed by $1 \mathrm{mcg} / \mathrm{kg} / \mathrm{hr}$ continuous infusion and then 100 mcg subcutaneous injections twice daily. As he continued to have bloody stools, the dose of subcutaneous octreotide was increased to 150 mcg three times daily. Bloody stools resolved and he was started on long-acting depot octreotide $10 \mathrm{mg}$ monthly injections. His 
hemoglobin stabilized and increased to the baseline (Figure 4). After two years without bleeding, octreotide was discontinued. He ultimately underwent heart transplant. Unfortunately, he died several years later due to complications of heart disease.

\section{Discussion:}

Gastrointestinal bleeding may be caused by various vascular anomalies like hemangiomas, angiodysplasias, gastric antral vascular ectasia (GAVE), radiation induced vascular ectasia and Dieulafoy's lesions. [1] Angiodysplasias (AD) are a common cause of occult as well as overt gastrointestinal bleeding in older patients. These may be asymptomatic or present with lifethreatening bleeding. These are, however, a rare cause of gastrointestinal bleeding in children. Histologically, AD are defined as abnormal, ectatic, dilated, tortuous and usually small $(<10$ $\mathrm{mm}$ ) blood vessels visualized within the mucosal and submucosal layers of the gastrointestinal tract. The affected vessels are lined by endothelium only with little or no smooth muscle. Most $\mathrm{AD}$ (54-81.9\%) are detected in the cecum and ascending colon. [2]

The etiology of $\mathrm{AD}$ is not completely understood. These lesions are thought to develop secondary to chronic low-grade intermittent obstruction of submucosal veins as in patients with heart conditions causing elevated right heart pressures. Increased levels of vascular endothelial growth factor leading to dysregulated angiogenesis may also play a role in their development.[2] Congenital $\mathrm{AD}$ are found in younger patients presenting with gastrointestinal bleeding with no underlying medical illness. Acquired AD has a higher association with chronic kidney disease, cardiovascular disease (especially aortic valve disease) and vWD. [3, 4]

Endoscopy is currently the mainstay for diagnosis of AD. Esophagogastroduodenoscopy, colonoscopy and small bowel enteroscopy are used diagnostically for upper gastrointestinal, colonic and small bowel AD, respectively. Wireless capsule endoscopy has emerged as the first line investigation for the small bowel lesions as it is safer and has a higher yield compared to other invasive procedures. [2]

The treatment of AD includes endoscopic therapy and pharmacologic therapy (somatostatin analogues, hormonal therapy and thalidomide). Somatostatin is a peptide secreted by gastrointestinal cells. It decreases bleeding via a number of potential mechanisms including reduced splanchnic blood flow, increased vascular resistance, inhibition of angiogenesis, inhibition of pepsin, gastric acid secretion and increased platelet aggregation.[5] Most of the literature published on the use of octreotide in $\mathrm{AD}$ is in adult patients. In a retrospective analysis of 98 patients with a history of bleeding due to $\mathrm{AD}$, all patients received octreotide $0.1 \mathrm{mg}$ tid for 28 days and, then long acting octreotide $20 \mathrm{mg}$ monthly, from day 14 for 6 months. There was a significant increase in the mean hemoglobin levels and the number of bleeding episodes, hospitalizations, patients requiring blood transfusions and units of transfused red cells 
significantly decreased amongst all patients over the two-year period. [6] In another study, 24 patients with refractory small bowel angiodysplasias were treated with $20 \mathrm{mg}$ of long acting octreotide for a minimum of three months. 83\% of patients had undergone APC (Argon Plasma Coagulation) with DBE (Double balloon enteroscopy) within a week prior to initiating octreotide therapy. The response was assessed by rates of rebleeding, hemoglobin levels and transfusion requirements. $70 \%$ patients had a complete response and 20\% patients had a partial response. Average hemoglobin rate increase from $9.19 \mathrm{~g} / \mathrm{dL}$ to $11.35 \mathrm{~g} / \mathrm{dL}$. [7] Long term efficacy of octreotide in prevention of rebleeding from AD was evaluated in a placebo controlled trial. 32 patients with bleeding from AD were treated with octreotide $50 \mathrm{mu} 12 \mathrm{~h}$ subcutaneously. These patients were followed for a period of 2 years. Treatment failure occurred in $23 \%$ of patients in the octreotide group and $48 \%$ in the placebo group. No significant differences between the groups were observed according to number of bleeding episodes.[8] A systematic review and meta-analysis of 22 clinical trials to examine the efficacy of different modalities for management of gastrointestinal angiodysplasia was published in 2014. 72 adult patients (in four studies) out of the 831 total patients included in the analysis received octreotide. These patients were refractory to endoscopic therapy. The pooled odds ratio was reported to be 14.5 for cessation of bleeding. [9] Side effects associated with octreotide therapy are usually mild or transient. These include diarrhea, nausea, vomiting, hyperglycemia, inhibition of gallbladder contractility.[10] Vitamin B12 deficiency has also been reported. In a prospective study, octreotide treatment was discontinued in 5 of 24 patients due to adverse events such as thrombocytopenia, choledocholithiasis and biliary sepsis, severe joint pains, severe injection site pain and acute blistering skin reaction, in five of twenty-four patients.[7] The simple route of administration and lack of major adverse events makes it a valuable potential alternative therapy to endoscopy. [11]

Other medical therapies have been studied for control of bleeding due to AD. An open label randomized trial investigated the efficacy of thalidomide for refractory bleeding over four months in 55 patients, 52 of whom were found to have AD. Response rate in the thalidomide and control groups were $71.4 \%$ and 3.7\%, respectively. [12] Hormonal therapy with estrogen and progesterone has also been tried. One double-blind randomized controlled trial assessed the long term efficacy of estrogen-progesterone therapy in 72 cirrhotic patients for recurrent bleeding from AD. No significant differences were found between the groups in terms of number of bleeding episodes and transfusion requirements over more than one year of follow up. Treatment failure occurred in $39 \%$ patients in the treatment group compared to $46 \%$ patients in the placebo group. [13]

Endoscopic treatment of AD has been utilized in the form of heat coagulation or APC. A systematic review in 2014 analyzed data from 63 studies with medical or endoscopic treatment of bleeding angiodysplasias and GAVE. This review found that the studies evaluating the endoscopic modalities have been retrospective with small number of patients, leading to insufficient evidence to support efficacy of any single endoscopic therapy.[14] 69 patients 
undergoing endoscopy with APC treatment were followed up for a mean period of 17.3 months in a retrospective study. The median number of treatment sessions was 1.49. In 46 patients requiring blood transfusion, the mean pre-treatment hemoglobin of $7.3 \mathrm{~g} / \mathrm{dL}$ increased to 9.3 $\mathrm{g} / \mathrm{dL}$. The median transfusion requirement decreased from 2.5 to 0.1 units of packed red blood cells after treatment. Rebleeding was observed in 33.3\% patients after a mean period of around 12 months of follow up. The overall survival without rebleeding was $50 \%$ and $42 \%$ at 1 and 2 years respectively. [15]

The lack of pediatric data on the use of octreotide in pediatric patients has limited its use in this population. It is not currently FDA approved for management of bleeding due to AD and is used as an off-label drug. [11] The patients in this report needed hospitalization due to gastrointestinal bleeding secondary to angiodysplasias. The bleeding stopped with stabilization of hemoglobin after initiating octreotide and patients did not have recurrence of bleeding. Given the published recurrence rate of bleeding and potential impact in these children, octreotide was continued for a prolonged period. The main limitations of this study are the small number of patients and the challenges associated with determining the dose and duration of the octreotide therapy. These patients had a desirable outcome after starting octreotide, although a causative relationship cannot be established. Given the limited therapeutic armamentarium and the potentially high morbidity of gastrointestinal bleeding in the cardiac population, identification of the potential use of octreotide in this setting is important. Larger scale studies are needed to determine the efficacy of octreotide in controlling gastrointestinal bleeding due to AD in pediatric patients.

\section{References:}

1. Gordon, F.H., A. Watkinson, and H. Hodgson, Vascular malformations of the gastrointestinal tract. Best Pract Res Clin Gastroenterol, 2001. 15(1): p. 41-58.

2. Sami, S.S., S.A. Al-Araji, and K. Ragunath, Review article: gastrointestinal angiodysplasia pathogenesis, diagnosis and management. Aliment Pharmacol Ther, 2014. 39(1): p. 15-34.

3. Randi, A.M., Endothelial dysfunction in von Willebrand disease: angiogenesis and angiodysplasia. Thromb Res, 2016. 141 Suppl 2: p. S55-8.

4. Holleran, G., et al., Small bowel angiodysplasia and novel disease associations: a cohort study. Scand J Gastroenterol, 2013. 48(4): p. 433-8.

5. Szilagyi, A. and M.P. Ghali, Pharmacological therapy of vascular malformations of the gastrointestinal tract. Can J Gastroenterol, 2006. 20(3): p. 171-8.

6. Nardone, G., et al., Long acting release-octreotide as "rescue" therapy to control angiodysplasia bleeding: A retrospective study of 98 cases. Dig Liver Dis, 2014. 46(8): p. 688-94.

7. Holleran, G., et al., Long-acting somatostatin analogues provide significant beneficial effect in patients with refractory small bowel angiodysplasia: Results from a proof of concept open label mono-centre trial. United European Gastroenterol J, 2016. 4(1): p. 70-6.

8. Junquera, F., et al., Long-term efficacy of octreotide in the prevention of recurrent bleeding from gastrointestinal angiodysplasia. Am J Gastroenterol, 2007. 102(2): p. 254-60.

9. Jackson, C.S. and L.B. Gerson, Management of gastrointestinal angiodysplastic lesions (GIADs): $a$ systematic review and meta-analysis. Am J Gastroenterol, 2014. 109(4): p. 474-83; quiz 484. 
10. Chan, M.M., et al., Octreotide: a drug often used in the critical care setting but not well understood. Chest, 2013. 144(6): p. 1937-45.

11. Iannone, A., et al., Gastrointestinal bleeding from vascular malformations: Is octreotide effective to rescue difficult-to-treat patients? Clin Res Hepatol Gastroenterol, 2016. 40(4): p. 373-7.

12. Ge, Z.Z., et al., Efficacy of thalidomide for refractory gastrointestinal bleeding from vascular malformation. Gastroenterology, 2011. 141(5): p. 1629-37.e1-4.

13. Junquera, F., et al., A multicenter, randomized, clinical trial of hormonal therapy in the prevention of rebleeding from gastrointestinal angiodysplasia. Gastroenterology, 2001. 121(5): p. 1073-9.

14. Swanson, E., et al., Medical and endoscopic therapies for angiodysplasia and gastric antral vascular ectasia: a systematic review. Clin Gastroenterol Hepatol, 2014. 12(4): p. 571-82.

15. Bizid, S., et al., Argon plasma coagulation in the management of symptomatic gastrointestinal angiodysplasia: experience in 69 consecutive patients. Tunis Med, 2015. 93(10): p. 606-11. 
Figure 1
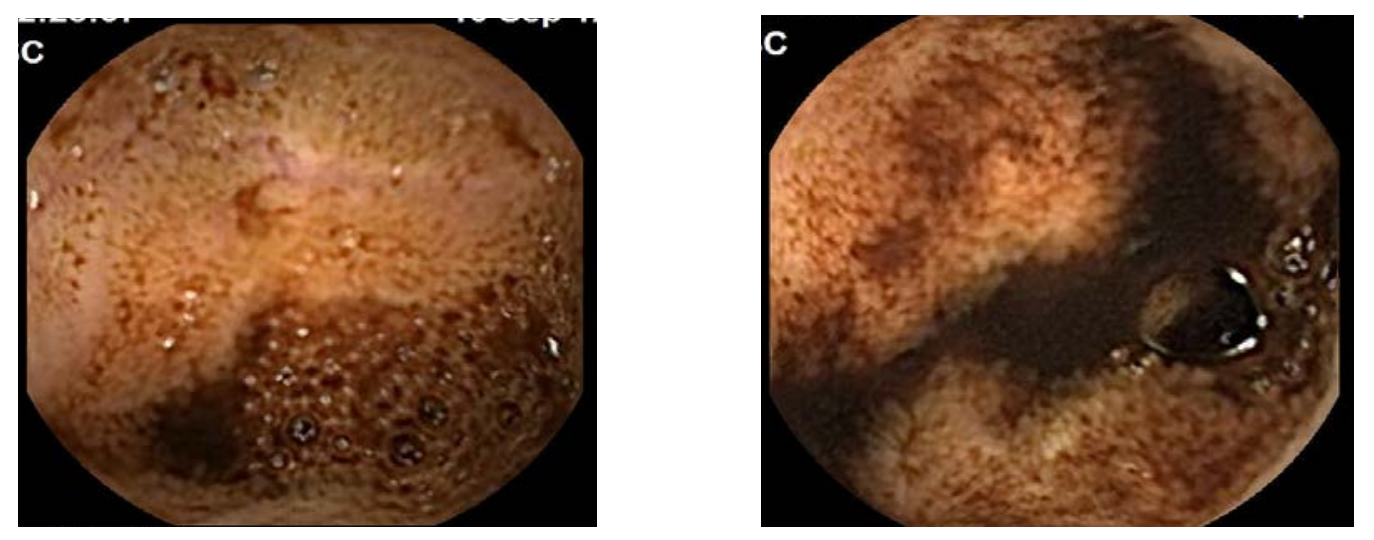

Upper endoscopy images of angiodysplasia, Patient A 
Figure 2- Hemoglobin trend, Patient A

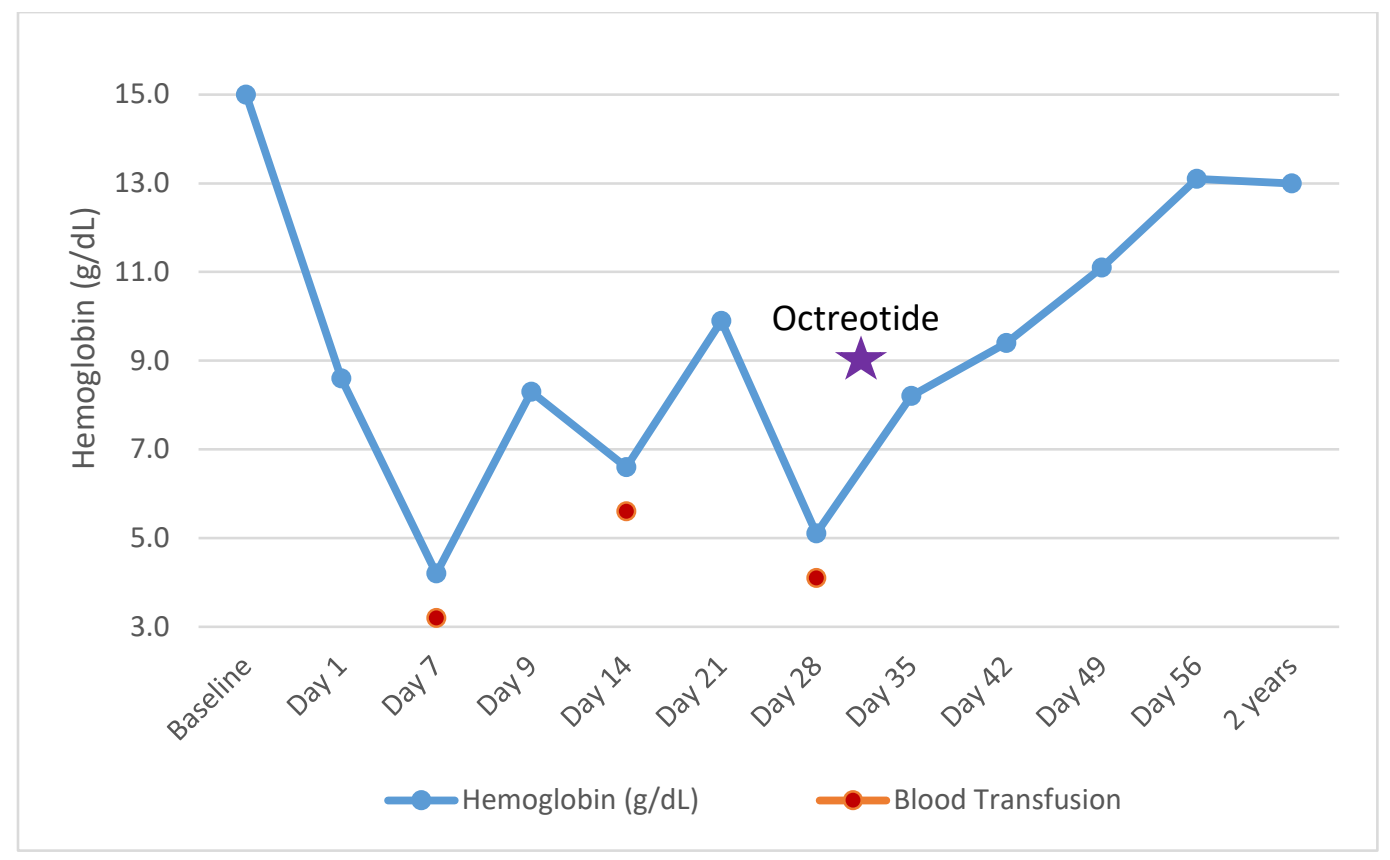

Blue solid line- Trend of hemoglobin

Red solid dots- Points in time when patient received blood transfusion

Star- Initiation of octreotide 
Figure 3

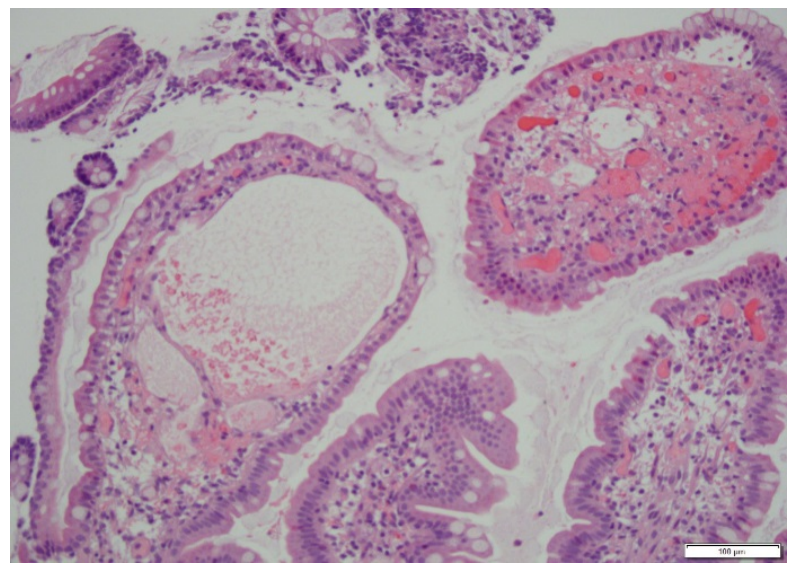

H\&E stain microscopic image of angiodysplasia on biopsy of Terminal Ileum, Patient B 
Figure 4- Hemoglobin trend, Patient B

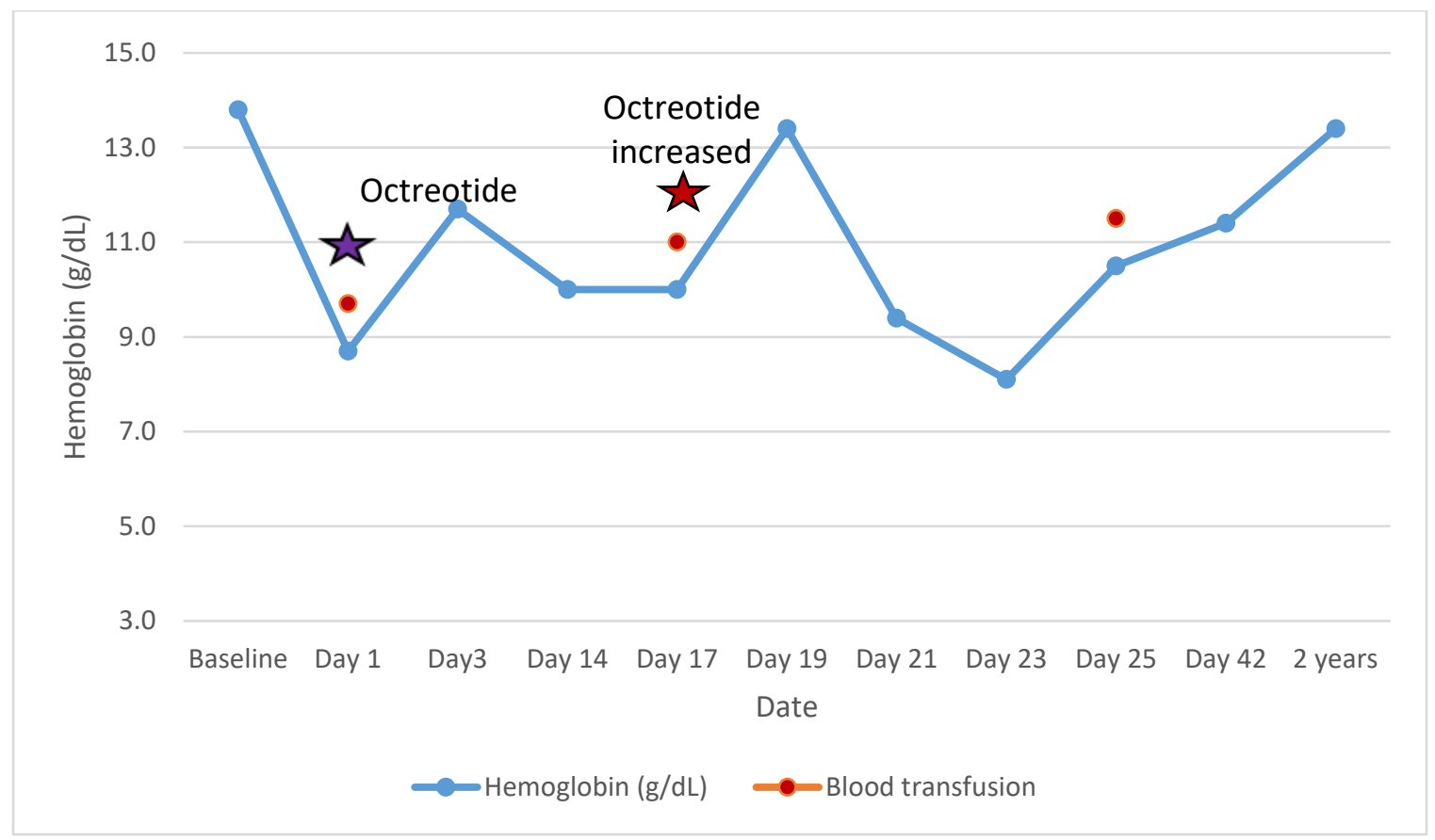

Blue solid line- Trend of hemoglobin

Red solid dots- Points in time when patient received blood transfusion

Purple Star- Initiation of octreotide

Red Star- Increase in dose of octreotide 